\title{
VISCOSITY APPROXIMATION METHOD FOR NONEXPANSIVE NONSELF-MAPPING AND VARIATIONAL INEQUALITY
}

\author{
ZHENHUA HE ${ }^{1 *}$, CAN CHEN ${ }^{1}$, AND FENG GU ${ }^{2}$
}

\begin{abstract}
Let $E$ be a real reflexive Banach space which has uniformly Gâteaux differentiable norm. Let $K$ be aclosed convex subset of $E$ which is also a sunny nonexpansive retract of $E$, and $T: K \rightarrow E$ be nonexpansive mapping satisfying the weakly inward condition and $F(T)=\{x \in K, T x=x\} \neq \emptyset$, and $f: K \rightarrow K$ be a contractive mapping. Suppose that $x_{0} \in K,\left\{x_{n}\right\}$ is defined by

$$
\left\{\begin{array}{l}
x_{n+1}=\alpha_{n} f\left(x_{n}\right)+\left(1-\alpha_{n}\right)\left((1-\delta) x_{n}+\delta y_{n}\right), \\
y_{n}=P\left(\beta_{n} x_{n}+\left(1-\beta_{n}\right) T x_{n}\right), \quad n \geq 0
\end{array}\right.
$$

where $\delta \in(0,1), \alpha_{n}, \beta_{n} \in[0,1], P$ is sunny nonexpansive retractive from $E$ into $K$. Under appropriate conditions, it is shown that $\left\{x_{n}\right\}$ converges strongly to a fixed point $T$ and the fixed point solutes some variational inequalities. The results in this paper extend and improve the corresponding results of [2] and some others.
\end{abstract}

\section{INTRODUCTION AND PRELIMINARIES}

Let $E$ be a real Banach space and $E^{*}$ its dual space. Let $J$ denote the normalized duality mapping from $E$ into $2^{E^{*}}$ defined by $J(x)=\left\{f \in E^{*}:\langle x, f\rangle=\|x\|^{2}=\right.$ $\left.\|f\|^{2}\right\}$, where $\langle\cdot, \cdot\rangle$ denote the generalized duality pairing between $E$ and $E^{*}$. It is well-known that if $E^{*}$ is strictly convex then $J$ is sing-valued. In the sequel, we shall denote the single-valued normalized duality mapping by $j$.

Date: Received: 19 December 2008.

* Corresponding author. The present studies were supported by the Honghe University foundation(XSS07006), the Scientific Research Foundation from Yunnan Province Education Committee (08Y0338).

2000 Mathematics Subject Classification. 47H09, 47H10, 47J05, 54H25.

Key words and phrases. Strong convergence, Nonexpansive nonself-mapping, Viscosity approximation method, Uniformly Gâteaux differentiable norm, Variational inequality. 
We first recall some definitions and conclusions:

Definition 1.1. $T$ is a mapping with domain $D(T)$ and $R(T)$ in $E . T$ is said to be a $L$-Lipschitz mapping, if $\forall x, y \in D(T),\|T x-T y\| \leq L\|x-y\|$. Especially, if $L=1$, i.e. $\|T x-T y\| \leq\|x-y\|$, then $T$ is said to non-expansive; if $0<L<1$, then $T$ is said to contraction mapping.

Definition 1.2. Let $K$ be a nonempty closed convex subsets of a Banach $E$. A mapping $P: E \rightarrow K$ is called a retraction from $E$ into $K$ if $P$ is continuous with $F(P)=\{x \in E: P x=x\}=K$. A mapping $P: E \rightarrow K$ is called sunny if

$$
P(P x+t(x-P x))=P x, \forall x \in E
$$

whenever $P x+t(x-P x) \in E$ and $\forall \in t>0$. A subset $K$ of $E$ is said to be a sunny nonexpansive retract of $E$ if there exists a sunny nonexpansive retraction from $E$ into $K$. For more details, see [4].

Let $S=\{x \in E:\|x\|=1\}$ denote the unit sphere of the real Banach space $E$. $E$ is said to have a Gâteaux differentiable norm if the limit

$$
\lim _{t \rightarrow 0} \frac{\|x+t y\|-\|x\|}{t}
$$

exists for each $x, y \in S$; and $E$ is said to have a uniformly Gâteaux differentiable norm if for each $y \in S$, the limit is attained uniformly for $x \in S$. Furthermore, if $E$ has a uniformly Gâteaux differentiable norm, then the duality map $j$ is normto-weak ${ }^{*}$ uniformly continuous on bounded subsets of $E$ (see, p.111 of [4]). Let $E$ be a normed space with $\operatorname{dim} E \geq 2$, the modulus of smoothness of $E$ is the function $\rho_{E}:[0, \infty) \rightarrow[0, \infty)$ defined by

$$
\rho_{E}(\tau):=\sup \left\{\frac{\|x+y\|+\|x-y\|}{2}-1:\|x\|=1 ;\|y\|=\tau\right\} .
$$

The space $E$ is called uniformly smooth if and only if $\lim _{\tau \rightarrow 0^{+}} \rho_{E} \tau / \tau=0$.

Let $F(T)$ denote a fixed point set of mapping $T$.

Let $K$ be a nonempty convex subset of a Banach space $E$. Then for $x \in K$, set $I_{K}(x)$ is called inward set $[2,7]$, where

$$
I_{K}(x)=\{y \in E: y=x+\lambda(z-x), z \in K \text { and } \lambda \geq 0\} .
$$

A mapping $T: K \rightarrow E$ is said to be satisfying the inward condition if $T x \in I_{K}(x)$ for all $x \in K$. T is also said to be satisfying the weakly inward condition if for each $x \in K, T x \in \overline{I_{K}(x)}\left(\overline{I_{K}(x)}\right.$ is the closure of $\left.I_{K}(x)\right)$. Clearly $K \subset I_{K}(x)$ and it is not hard to show that $I_{K}(x)$ is a convex set as $K$ does.

Let $K$ be a close convex subset of a uniformly smooth Banach space $E, f$ : $K \rightarrow K$ a contraction, $T: K \rightarrow K$ a nonexpansive mapping with $F(T) \neq \emptyset$. Then for any $t \in(0,1)$, the mapping

$$
T_{t}^{f}: x \mapsto t f(x)+(1-t) T x
$$

is also contraction. Let $x_{t}$ denote the unique fixed point of $T_{t}^{f}$. In [6], H.K.Xu proved that as $t \downarrow 0,\left\{x_{t}\right\}$ converges to a fixed point $u$ of $T$ that is the unique solution of the variational inequality

$$
\langle(I-f) u, j(u-p)\rangle \leq 0 \text { for all } p \in F(T) .
$$


H.K. Xu also proved the following explicit iterative process $\left\{x_{n}\right\}$ given by

$$
x_{n+1}=\alpha_{n} f\left(x_{n}\right)+\left(1-\alpha_{n}\right) T x_{n}
$$

converges strongly to a fixed point $p$ of $T$.

Let $K$ be a close convex subset of a real Banach space $E$ which is also a sunny nonexpansive retract of $E . \quad f: K \rightarrow K$ is a contraction. $T: K \rightarrow E$ is a nonexpansive nonself-mapping. Inspired by Xu [6], in 2006, Y.Song and R.Chen [2] considered the following algorithm,

$$
x_{n+1}=P\left(\alpha_{n} f\left(x_{n}\right)+\left(1-\alpha_{n}\right) T x_{n}\right), n \geq 0,
$$

where $x_{0} \in K, P$ is a sunny nonexpansive retractive from $E$ into $K, \alpha_{n} \in(0,1)$. Then Y.Song and R. Chen [2] obtained the following results:

Theorem 1.3. (Theorem 2.4 of [2]). Let E be a reflexive Banach space which admits a weakly sequentially continuous $J$ from $E$ to $E^{*}$. Suppose $K$ is a nonempty closed convex subset of $E$ which is also a sunny nonexpansive retract of $E$, and $T: K \rightarrow E$ is a nonexpansive mapping satisfying the weakly inward condition and $F(T) \neq \emptyset$. Let $\left\{x_{n}\right\}$ be defined by (1.1), where $P$ is a sunny nonexpansive retract from $E$ into $K$, and $\alpha_{n} \in(0,1)$ satisy the following conditions:

(i) $\alpha_{n} \rightarrow 0$, as $n \rightarrow \infty$, and $\sum_{n=1}^{\infty} \alpha_{n}=\infty$

(ii) either $\sum_{n=0}^{\infty}\left|\alpha_{n+1}-\alpha_{n}\right|<\infty$ or $\lim _{n \rightarrow \infty} \frac{\alpha_{n}}{\alpha_{n+1}}=1$.

Then $\left\{x_{n}\right\}$ converges strongly to a fixed point $p$ of $T$ such that $p$ is the unique solution in $F(T)$ to the following variational inequality:

$$
\langle(I-f) p, j(p-u)\rangle \leq 0, \quad \forall u \in F(T) .
$$

Motivated by Song and Chen's work, in this paper, we introduce a new composite iterative scheme as follows:

$$
\left\{\begin{array}{l}
x_{n+1}=\alpha_{n} f\left(x_{n}\right)+\left(1-\alpha_{n}\right)\left((1-\delta) x_{n}+\delta y_{n}\right), \\
y_{n}=P\left(\beta_{n} x_{n}+\left(1-\beta_{n}\right) T x_{n}\right), \quad n \geq 0
\end{array}\right.
$$

where $\alpha_{n}, \beta_{n} \in(0,1), \sigma \in(0,1)$ is arbitrary (but fixed). Under appropriate conditions, the $\left\{x_{n}\right\}$ defined by (1.2) converges strongly to a fixed point $q$ of $T$ such that $q$ is a solution of some variational inequalities. The results obtained in this paper extend and improve the corresponding that of [2] and some others. At the same time, this paper provides a new approach for the construction of a fixed point of nonexpansive mapping.

In what follows, we shall make use of the following Lemmas.

Lemma 1.4. ([1]). Let $E$ be a real normed linear space and $J$ the normalized duality mapping on $E$, then for each $x, y \in E$ and $j(x+y) \in J(x+y)$, we have

$$
\|x+y\|^{2} \leq\|x\|^{2}+2\langle y, j(x+y)\rangle .
$$

Lemma 1.5. (Suzuki, [3]).Let $\left\{x_{n}\right\}$ and $\left\{y_{n}\right\}$ be bounded sequences in a Banach space $E$ and let $\left\{\beta_{n}\right\}$ be a sequence in $[0,1]$ with $0<\liminf _{n \rightarrow \infty} \beta_{n} \leq$ $\limsup \sup _{n \rightarrow \infty} \beta_{n}<1$. Suppose $x_{n+1}=\beta_{n} y_{n}+\left(1-\beta_{n}\right) x_{n}$ for all integers $n \geq 0$ and $\lim \sup _{n \rightarrow \infty}\left(\left\|y_{n+1}-y_{n}\right\|-\left\|x_{n+1}-x_{n}\right\|\right) \leq 0$, then, $\lim _{n \rightarrow \infty}\left\|y_{n}-x_{n}\right\|=0$. 
Lemma 1.6. ([8]). Let $\left\{a_{n}\right\}$ be a sequence of nonnegative real numbers satisfying the following relation:

$$
a_{n+1} \leq\left(1-\alpha_{n}\right) a_{n}+\alpha_{n} \sigma_{n}+\gamma_{n}, n \geq 0,
$$

if (i) $\alpha_{n} \in[0,1], \sum \alpha_{n}=\infty$; (ii) $\lim \sup \sigma_{n} \leq 0$; (iii) $\gamma_{n} \geq 0, \sum \gamma_{n}<\infty$, then $a_{n} \rightarrow 0$, as $n \rightarrow \infty$.

Let $\mu$ be a continuous linear functional on $l^{\infty}$ satisfying $\|\mu\|=1=\mu(1)$. Then we know that $\mu$ is a mean on $N$ if and only if

$$
\inf \left\{a_{n} ; n \in N\right\} \leq \mu(a) \leq \sup \left\{a_{n} ; n \in N\right\}
$$

for every $a=\left(a_{1}, a_{2}, \ldots\right) \in l^{\infty}$. According to time and circumstances, we use $\mu_{n}\left(a_{n}\right)$ instead of $\mu(a)$. A mean $\mu$ on $N$ is called a Banach limit if $\mu_{n}\left(a_{n}\right)=$ $\mu_{n}\left(a_{n+1}\right)$ for every $a=\left(a_{1}, a_{2}, \ldots\right) \in l^{\infty}$. Furthermore, we know the following result [5, Lemma 1] and[4, Lemma4.5.4].

Lemma 1.7. ([5], Lemma 1). Let $K$ be a nonempty closed convex subset of a Banach space $E$ with a uniformly Gâteaux differentiable norm. Let $\left\{x_{n}\right\}$ be a bounded sequence of $E$ and let $\mu$ be a mean on $N$. Let $z \in K$. Then

$$
\mu_{n}\left\|x_{n}-z\right\|=\min _{y \in K} \mu_{n}\left\|x_{n}-y\right\|
$$

if and only if

$$
\mu_{n}\left\langle y-z, j\left(x_{n}-z\right)\right\rangle \leq 0, \quad \forall y \in K
$$

where $j$ is the duality mapping of $E$.

Lemma 1.8. (Lemma 1.2 of [2]). Let $E$ be a smooth Banach space, and $K$ be a nonempty closed convex subset of $E$ which is also a sunny nonexpansive retract of $E$, and $T: K \rightarrow E$ be mapping satisfying the weakly inward condition, and $P$ be a sunny nonexpansive retraction from $E$ into $K$. Then $F(T)=F(P T)$.

\section{MAin RESUlts}

Throughout this paper, suppose that

(a) $E$ is a real reflexive Banach space $E$ which has a uniformly Gâteaux differentiable norms;

(b) $K$ is a nonempty closed convex subset of $E$;

(c) every nonempty closed bounded convex subset of $E$ has the fixed point property for nonexpansive mappings.

Lemma 2.1. Let $T: K \rightarrow K$ be a nonexpansive mapping with $F(T)=\{x \in K$ : $T x=x\} \neq \emptyset$. Let $f: K \rightarrow K$ be a contraction with contraction constant $\in$ $(0,1)$, then there exists $x_{t} \in K$ such that

$$
x_{t}=t f\left(x_{t}\right)+(1-t) T x_{t},
$$

where $t \in(0,1)$. Further, as $t \rightarrow 0^{+}, x_{t}$ converges strongly a fixed point $q \in F(T)$ which solutes the following variational inequality:

$$
\langle q-f(q), j(q-p)\rangle \leq 0, \quad \forall p \in F(T) .
$$


Proof. Firstly, let $H_{t}^{f}$ denote a mapping defined by

$$
H_{t}^{f} x=t f(x)+(1-t) T x, \forall t \in(0,1), \forall x \in E .
$$

Obviously, $H_{t}^{f}$ is contraction, then by Banach contraction mapping principle there exists $x_{t} \in K$ such that

$$
x_{t}=t f\left(x_{t}\right)+(1-t) T x_{t} .
$$

Now, let $q \in F(T)$, then

$\left\|x_{t}-q\right\|=\left\|t\left(f\left(x_{t}\right)-q\right)+(1-t)\left(T x_{t}-q\right)\right\| \leq(1-t+t \alpha)\left\|x_{t}-q\right\|+t\|f(q)-q\|$, i.e.,

$$
\left\|x_{t}-q\right\| \leq \frac{\|f(q)-q\|}{1-\alpha} .
$$

Hence $\left\{x_{t}\right\}$ is bounded. Assume that $t_{n} \rightarrow 0^{+}$as $n \rightarrow \infty$. Set $x_{n}:=x_{t_{n}}$, define a function $g$ on $K$ by

$$
g(x)=\mu_{n}\left\|x_{n}-x\right\|^{2} .
$$

Let

$$
C=\left\{x \in K ; g(x)=\min _{y \in E} \mu_{n}\left\|x_{n}-y\right\|^{2}\right\} .
$$

It is easy to see that $C$ is a closed convex bounded subset of $K$. Since $\| x_{n}-$ $T x_{n} \| \rightarrow 0(n \rightarrow \infty)$, hence

$$
g(T x)=\mu_{n}\left\|x_{n}-T x\right\|^{2}=\mu_{n}\left\|T x_{n}-T x\right\|^{2} \leq \mu_{n}\left\|x_{n}-x\right\|^{2}=g(x),
$$

it follows that $T(C) \subset C$, that is $C$ is invariant under $T$. By assumption (c), non-expansive mapping $T$ has fixed point $q \in C$. Using Lemma 1.7 we obtain

$$
\mu_{n}\left\langle x-q, j\left(x_{n}-q\right)\right\rangle \leq 0 .
$$

Taking $x=f(q)$, then

$$
\mu_{n}\left\langle f(q)-q, j\left(x_{n}-q\right)\right\rangle \leq 0
$$

Since

$$
x_{t}-q=t\left(f\left(x_{t}\right)-q\right)+(1-t)\left(T x_{t}-q\right),
$$

then

$$
\begin{aligned}
\left\|x_{t}-q\right\|^{2} & =t\left\langle f\left(x_{t}\right)-q, j\left(x_{t}-q\right)\right\rangle+(1-t)\left\langle T x_{t}-q, j\left(x_{t}-q\right)\right\rangle \\
& \leq t\left\langle f\left(x_{t}\right)-q, j\left(x_{t}-q\right)\right\rangle+(1-t)\left\|x_{t}-q\right\|^{2}
\end{aligned}
$$

Further,

$$
\begin{aligned}
\left\|x_{t}-q\right\|^{2} & \leq\left\langle f\left(x_{t}\right)-q, j\left(x_{t}-q\right)\right\rangle \\
& =\left\langle f\left(x_{t}\right)-f(q), j\left(x_{t}-q\right)\right\rangle+\left\langle f(q)-q, j\left(x_{t}-q\right)\right\rangle .
\end{aligned}
$$

Thus,

$$
\mu_{n}\left\|x_{n}-q\right\|^{2} \leq \mu_{n} \alpha\left\|x_{n}-q\right\|^{2}+\mu_{n}\left\langle f(q)-q, j\left(x_{n}-q\right)\right\rangle .
$$

it follows from (2.3) that

$$
\mu_{n}\left\|x_{n}-q\right\|^{2}=0
$$

Hence there exists a subsequence of $\left\{x_{n}\right\}$ which is still denoted by $\left\{x_{n}\right\}$ such that $\lim _{n \rightarrow \infty}\left\|x_{n}-q\right\|=0$. Now assume that another subsequence $\left\{x_{m}\right\}$ of $\left\{x_{n}\right\}$ converge strongly to $\bar{q} \in F(T)$. Since $j$ is norm-to-weak $k^{*}$ uniformly continuous on bounded subsets of $E$, then for any $p \in F(T)$,we have 


$$
\begin{aligned}
\left|\left\langle x_{m}-f\left(x_{m}\right), j\left(x_{m}-p\right)\right\rangle-\langle\bar{q}-f(\bar{q}), j(\bar{q}-p)\rangle\right| \\
=\mid\left\langle x_{m}-f\left(x_{m}\right)-(\bar{q}-f(\bar{q})), j\left(x_{m}-p\right)\right\rangle \\
\quad+\left\langle(\bar{q}-f(\bar{q})), j\left(x_{m}-p\right)\right\rangle-\langle\bar{q}-f(\bar{q}), j(\bar{q}-q)\rangle \mid \\
\leq \quad\left\|(I-f) x_{m}-(I-f) \bar{q}\right\|\left\|x_{m}-p\right\| \\
\quad+\left|\left\langle\bar{q}-f(\bar{q}), j\left(x_{m}-p\right)-j(\bar{q}-p)\right\rangle\right| \rightarrow 0(m \rightarrow \infty),
\end{aligned}
$$

i.e.,

$$
\langle\bar{q}-f(\bar{q}), j(\bar{q}-p)\rangle=\lim _{n \rightarrow \infty}\left\langle x_{m}-f\left(x_{m}\right), j\left(x_{m}-p\right)\right\rangle .
$$

Since $x_{m}=t f\left(x_{m}\right)+(1-t) T x_{m}$, we have

$$
(I-f) x_{m}=-\frac{1-t}{t}(I-T) x_{m},
$$

hence for any $p \in F(T)$,

$$
\left\langle(I-f) x_{m}, j\left(x_{m}-p\right)\right\rangle=-\frac{1-t}{t}\left\langle(I-T) x_{m}-(I-T) p, j\left(x_{m}-p\right)\right\rangle \leq 0,
$$

it follows from (2.5) and (2.6) that

$$
\langle\bar{q}-f(\bar{q}), j(\bar{q}-p)\rangle \leq 0 .
$$

Interchange $p$ and $q$ to obtain

$$
\langle\bar{q}-f(\bar{q}), j(\bar{q}-q)\rangle \leq 0,
$$

i.e.,

$$
\langle\bar{q}-q+q-f(\bar{q}), j(\bar{q}-q)\rangle \leq 0,
$$

hence

$$
\|\bar{q}-q\|^{2} \leq\langle f(\bar{q})-q, j(\bar{q}-q)\rangle .
$$

Interchange $q$ and $\bar{q}$ to obtain

$$
\|\bar{q}-q\|^{2} \leq\langle f(q)-\bar{q}, j(q-\bar{q})\rangle .
$$

Adding up (2.10) and (2.11) yields that

$$
2\|\bar{q}-q\|^{2} \leq(1+\alpha)\|\bar{q}-q\|,
$$

this implies that $q=\bar{q}$. Hence $x_{t} \rightarrow q$ as $t \rightarrow 0^{+}$and $q$ is a solution of the following variational inequality

$$
\langle q-f(q), j(q-p)\rangle \leq 0, \quad \forall p \in \mathrm{F}(\mathrm{T}) .
$$

This completes the proof of Lemma 2.1.

Theorem 2.2. Let $K$ be a sunny nonexpansive retract of $E$. $T: K \rightarrow E$ is a nonexpansive mapping satisfying the weakly inward condition and $F(T) \neq \emptyset$. $f: K \rightarrow K$ is contractive with constant $\alpha \in(0,1)$. Let $P$ be a sunny nonexpansive retraction from $E$ into $K$. For given $x_{0} \in K$, let $\left\{x_{n}\right\}$ be generated by the algorithm

$$
\left\{\begin{array}{l}
x_{n+1}=\alpha_{n} f\left(x_{n}\right)+\left(1-\alpha_{n}\right)\left((1-\delta) x_{n}+\delta y_{n}\right), \\
y_{n}=P\left(\beta_{n} x_{n}+\left(1-\beta_{n}\right) T x_{n}\right), \quad n \geq 0,
\end{array}\right.
$$

where $\left\{\alpha_{n}\right\},\left\{\beta_{n}\right\} \subset[0,1] . \quad \delta \in(0,1)$ is arbitrary (but fixed). Suppose that $\left\{\alpha_{n}\right\},\left\{\beta_{n}\right\}$ satisfy the following conditions:

(i) $\alpha_{n} \rightarrow 0$ as $n \rightarrow \infty, \Sigma_{n=0}^{\infty} \alpha_{n}=\infty$, 
(ii) $0 \leq \beta_{n}<a,\left|\beta_{n+1}-\beta_{n}\right| \rightarrow 0$ as $n \rightarrow \infty$, where $a \in(0,1)$.

Then $\left\{x_{n}\right\}$ converges strongly to a fixed point $q \in F(T)$, where $q=\lim _{t \rightarrow 0^{+}} x_{t}$ is a solution of variational inequality (2.2).

Proof. We splits four steps to prove it.

Step 1. $\left\{x_{n}\right\}$ is bounded. In deed, by (2.13), it is easy to see that

$$
\begin{aligned}
\left\|y_{n}-x^{*}\right\| & =\left\|P\left(\beta_{n} x_{n}+\left(1-\beta_{n}\right) T x_{n}\right)-x^{*}\right\|=\left\|P\left(\beta_{n} x_{n}+\left(1-\beta_{n}\right) T x_{n}\right)-P x^{*}\right\| \\
& \leq\left\|\beta_{n}\left(x_{n}-x^{*}\right)+\left(1-\beta_{n}\right)\left(T x_{n}-x^{*}\right)\right\| \leq\left\|x_{n}-x^{*}\right\|
\end{aligned}
$$

and

$$
\begin{aligned}
\left\|x_{n+1}-x^{*}\right\|= & \left\|\alpha_{n}\left(f\left(x_{n}\right)-x^{*}\right)+\left(1-\alpha_{n}\right)\left((1-\delta)\left(x_{n}-x^{*}\right)+\delta\left(y_{n}-x^{*}\right)\right)\right\| \\
\leq & \left(1-\alpha_{n}\right)(1-\delta)\left\|x_{n}-x^{*}\right\|+\alpha_{n} \alpha\left\|x_{n}-x^{*}\right\| \\
& +\alpha_{n}\left\|f\left(x^{*}\right)-x^{*}\right\|+\left(1-\alpha_{n}\right) \delta\left\|y_{n}-x^{*}\right\|,
\end{aligned}
$$

where $x^{*} \in F(T)$. It follows from (2.14) and (2.15) that

$$
\left\|x_{n+1}-x^{*}\right\| \leq\left(1-(1-\alpha) \alpha_{n}\right)\left(\left\|x_{n}-x^{*}\right\|+\alpha_{n}\left\|f\left(x^{*}\right)-x^{*}\right\| .\right.
$$

By simplicity deducing, from (2.16) we have

$$
\left\|x_{n}-x^{*}\right\| \leq \max \left\{\left\|x_{0}-x^{*}\right\|, \frac{\left\|f\left(x^{*}\right)-x^{*}\right\|}{1-\alpha}\right\}, \quad n \geq 0 .
$$

Hence, $\left\{x_{n}\right\}$ is bounded and so is $\left\{y_{n}\right\}$.

Step 2. $\left\|x_{n+1}-x_{n}\right\| \rightarrow 0$ as $n \rightarrow \infty$. In deed, let $M>0$ be a constant such that $\max \left\{\left\|x_{n+1}\right\|,\left\|x_{n}\right\|,\left\|y_{n+1}\right\|,\left\|T x_{n+1}\right\|,\left\|T x_{n}\right\|,\left\|f\left(x_{n+1}\right)\right\|,\left\|f\left(x_{n}\right)\right\|\right\} \leq M$.

It follows from (2.13) that

$$
\begin{aligned}
\left\|y_{n+1}-y_{n}\right\| & =\left\|P\left(\beta_{n+1} x_{n+1}+\left(1-\beta_{n+1}\right) T x_{n+1}\right)-P\left(\beta_{n} x_{n}+\left(1-\beta_{n}\right) T x_{n}\right)\right\| \\
& \leq\left\|\beta_{n+1} x_{n+1}-\beta_{n} x_{n}\right\|+\left\|\left(1-\beta_{n+1}\right) T x_{n+1}-\left(1-\beta_{n}\right) T x_{n}\right\| \\
& \leq 2\left|\beta_{n+1}-\beta_{n}\right| M+\left\|x_{n+1}-x_{n}\right\| .
\end{aligned}
$$

Now, let $\gamma_{n}=\delta+\alpha_{n}(1-\delta), \bar{y}_{n}=\frac{x_{n+1}-x_{n}+\gamma_{n} x_{n}}{\gamma_{n}}=\frac{\alpha_{n} f\left(x_{n}\right)+\left(1-\alpha_{n}\right) \delta y_{n}}{\gamma_{n}}$, then

$$
\begin{aligned}
& \bar{y}_{n+1}-\bar{y}_{n} \\
& =\frac{\alpha_{n+1}}{\gamma_{n+1}} f\left(x_{n+1}\right)-\frac{\alpha_{n}}{\gamma_{n}} f\left(x_{n}\right)+\frac{\left(1-\alpha_{n+1}\right) \delta y_{n+1}}{\gamma_{n+1}}-\frac{\left(1-\alpha_{n}\right) \delta y_{n}}{\gamma_{n}} \\
& =\frac{\alpha_{n+1}}{\gamma_{n+1}} f\left(x_{n+1}\right)-\frac{\alpha_{n}}{\gamma_{n}} f\left(x_{n}\right)+\frac{\left(1-\alpha_{n}\right) \delta}{\gamma_{n}}\left(y_{n+1}-y_{n}\right)+\left(\frac{1-\alpha_{n+1}}{\gamma_{n+1}}-\frac{1-\alpha_{n}}{\gamma_{n}}\right) \delta y_{n+1},
\end{aligned}
$$

which yields that

$$
\left\|\bar{y}_{n+1}-\bar{y}_{n}\right\| \leq 2 \frac{\alpha_{n+1}+\alpha_{n}}{\gamma_{n+1} \gamma_{n}} M+\frac{\left(1-\alpha_{n}\right) \delta}{\gamma_{n}}\left\|y_{n+1}-y_{n}\right\| .
$$

It follows from (2.17) and (2.18) that

$$
\left\|\bar{y}_{n+1}-\bar{y}_{n}\right\| \leq 2 \frac{\alpha_{n+1}+\alpha_{n}}{\gamma_{n+1} \gamma_{n}} M+\frac{2\left|\beta_{n+1}-\beta_{n}\right| M}{\gamma_{n}}+\frac{\left(1-\alpha_{n}\right) \delta}{\gamma_{n}}\left\|x_{n+1}-x_{n}\right\| .
$$

Using the conditions (i-ii), from (2.19) we get that

$$
\limsup _{n \rightarrow \infty}\left\{\left\|\bar{y}_{n+1}-\bar{y}_{n}\right\|-\left\|x_{n+1}-x_{n}\right\|\right\} \leq 0 .
$$


Based on Lemma 1.5 and (2.20), we obtain $\lim _{n \rightarrow \infty}\left\|\bar{y}_{n}-x_{n}\right\|=0$, which implies $\lim _{n \rightarrow \infty}\left\|x_{n+1}-x_{n}\right\|=0$.

Step3. $\left\|x_{n}-P T x_{n}\right\| \rightarrow 0$ as $n \rightarrow \infty$. Since

$$
\left\|x_{n+1}-\left((1-\delta) x_{n}+\delta y_{n}\right)\right\|=\alpha_{n}\left\|f\left(x_{n}\right)-\left((1-\delta) x_{n}+\delta y_{n}\right)\right\| \rightarrow 0(n \rightarrow \infty)
$$

and

$\delta\left\|x_{n}-y_{n}\right\|-\left\|x_{n+1}-x_{n}\right\| \leq\left\|x_{n+1}-x_{n}-\delta\left(y_{n}-x_{n}\right)\right\|=\left\|x_{n+1}-\left((1-\delta) x_{n}+\delta y_{n}\right)\right\|$, hence,

$$
\left\|x_{n}-y_{n}\right\| \leq \frac{\left\|x_{n+1}-x_{n}\right\|+\left\|x_{n+1}-\left((1-\delta) x_{n}+\delta y_{n}\right)\right\|}{\delta} \rightarrow 0(n \rightarrow \infty) .
$$

Further,

$$
\left\|x_{n}-P T x_{n}\right\| \leq\left\|x_{n}-y_{n}\right\|+\left\|y_{n}-P T x_{n}\right\| \leq\left\|x_{n}-y_{n}\right\|+a\left\|x_{n}-P T x_{n}\right\|,
$$

which yields that

$$
\left\|x_{n}-P T x_{n}\right\| \rightarrow 0(n \rightarrow \infty) .
$$

Step 4. $\left\|x_{n}-x^{*}\right\| \rightarrow 0$ as $n \rightarrow \infty$, where $x^{*} \in F(T)$ and $x^{*}$ satisfies the variational inequality (2.2).

Since $P T$ is nonexpansive mapping, then by Lemma 2.1 there exists $x_{t}$ such that

$$
x_{t}=t f\left(x_{t}\right)+(1-t) P T x_{t}, \quad \forall t \in(0,1),
$$

Then, using Lemma 1.4, we have

$$
\begin{aligned}
& \left\|x_{t}-x_{n}\right\|^{2}=\left\|t\left(f\left(x_{t}\right)-x_{n}\right)+(1-t)\left(P T x_{t}-x_{n}\right)\right\|^{2} \\
& \leq(1-t)^{2}\left\|P T x_{t}-x_{n}\right\|^{2}+2 t\left\langle f\left(x_{t}\right)-x_{n}, j\left(x_{t}-x_{n}\right)\right\rangle \\
& \leq(1-t)^{2}\left(\left\|P T x_{t}-P T x_{n}\right\|+\left\|P T x_{n}-x_{n}\right\|\right)^{2}+2 t\left\langle f\left(x_{t}\right)-x_{t}+x_{t}-x_{n}, j\left(x_{t}-x_{n}\right)\right\rangle \\
& \leq\left(1+t^{2}\right)\left\|x_{t}-x_{n}\right\|^{2}+\left\|P T x_{n}-x_{n}\right\|\left(2\left\|x_{t}-x_{n}\right\|+\left\|P T x_{n}-x_{n}\right\|\right) \\
& \quad+2 t\left\langle f\left(x_{t}\right)-x_{t}, j\left(x_{t}-x_{n}\right)\right\rangle,
\end{aligned}
$$

hence,

$\left\langle f\left(x_{t}\right)-x_{t}, j\left(x_{n}-x_{t}\right)\right\rangle \leq \frac{t}{2}\left\|x_{t}-x_{n}\right\|^{2}+\frac{\left\|P T x_{n}-x_{n}\right\|}{2 t}\left(2\left\|x_{t}-x_{n}\right\|+\left\|P T x_{n}-x_{n}\right\|\right)$,

let $n \rightarrow \infty$ in the last inequality, then we obtain

$$
\limsup _{n \rightarrow \infty}\left\langle f\left(x_{t}\right)-x_{t}, j\left(x_{n}-x_{t}\right)\right\rangle \leq \frac{t}{2} M^{\prime},
$$

where $M^{\prime} \geq 0$ is a constant such that $\left\|x_{t}-x_{n}\right\|^{2} \leq M^{\prime}$ for all $t \in(0,1)$ and $n \geq 0$. Now letting $t \rightarrow 0^{+}$, then we have that

$$
\limsup _{t \rightarrow 0^{+}} \limsup _{n \rightarrow \infty}\left\langle f\left(x_{t}\right)-x_{t}, j\left(x_{n}-x_{t}\right)\right\rangle \leq 0 .
$$

Thus, for $\forall \varepsilon>0$, there exists a positive number $\delta^{\prime}$ such that for any $t \in\left(0, \delta^{\prime}\right)$,

$$
\limsup _{n \rightarrow \infty}\left\langle f\left(x_{t}\right)-x_{t}, j\left(x_{n}-x_{t}\right)\right\rangle \leq \frac{\varepsilon}{2} .
$$

On the other hand, By Lemma 1.8 and Lemma 2.1 we have $x_{t} \rightarrow x^{*} \in F(P T)=$ $F(T)$ as $t \rightarrow 0^{+}$. In addition, $j$ is norm-to-weak ${ }^{*}$ uniformly continuous on bounded subsets of $E$, so there exists $\delta^{\prime \prime}>0$ such that, for any $t \in\left(0, \delta^{\prime \prime}\right)$, we have 
$\mid\left\langle\left(f\left(x^{*}\right)-x^{*}, j\left(x_{n}-x^{*}\right)\right\rangle-\left\langle f\left(x_{t}\right)-x_{t}, j\left(x_{n}-x_{t}\right)\right\rangle\right|$

$\leq\left|\left\langle f\left(x^{*}\right)-x^{*}, j\left(x_{n}-x^{*}\right)-j\left(x_{n}-x_{t}\right)\right\rangle+\right|\left\langle f\left(x^{*}\right)-x^{*}, j\left(x_{n}-x_{t}\right)\right\rangle-\left\langle f\left(x_{t}\right)-x_{t}, j\left(x_{n}-x_{t}\right)\right\rangle \mid$

$\leq\left\|f\left(x^{*}\right)-x^{*}\right\|\left\|j\left(x_{n}-x^{*}\right)-j\left(x_{n}-x_{t}\right)\right\|+(1+\alpha)\left\|x_{t}-x^{*}\right\|\left\|x_{n}-x_{t}\right\|$

$<\frac{\varepsilon}{2}$.

Taking $\delta=\min \left\{\delta^{\prime}, \delta^{\prime \prime}\right\}$, for $t \in(0, \delta)$, we have that

$$
\left\langle f\left(x^{*}\right)-x^{*}, j\left(x_{n}-x^{*}\right)\right\rangle \leq\left\langle f\left(x_{t}\right)-x_{t}, j\left(x_{n}-x_{t}\right)\right\rangle+\frac{\varepsilon}{2} \text {. }
$$

Hence,

$$
\limsup _{n \rightarrow \infty}\left\langle f\left(x^{*}\right)-x^{*}, j\left(x_{n}-x^{*}\right)\right\rangle \leq \varepsilon, \text { where } \varepsilon>0 \text { is arbitrary, }
$$

which yields that

$$
\limsup _{n \rightarrow \infty}\left\langle f\left(x^{*}\right)-x^{*}, j\left(x_{n}-x^{*}\right)\right\rangle \leq 0 .
$$

Now we prove that $\left\{x_{n}\right\}$ converges strongly to $x^{*}$. It follows from Lemma 1.4 and (2.13) that

$$
\begin{aligned}
& \left\|x_{n+1}-x^{*}\right\|^{2}=\left\|\alpha_{n}\left(f\left(x_{n}\right)-x^{*}\right)+\left(1-\alpha_{n}\right)\left((1-\delta)\left(x_{n}-x^{*}\right)+\delta\left(y_{n}-x^{*}\right)\right)\right\|^{2} \\
& \leq\left(1-\alpha_{n}\right)^{2}\left\|(1-\delta)\left(x_{n}-x^{*}\right)+\delta\left(y_{n}-x^{*}\right)\right\|^{2}+2 \alpha_{n}\left\langle f\left(x_{n}\right)-x^{*}, j\left(x_{n+1}-x^{*}\right)\right\rangle \\
& =\left(1-\alpha_{n}\right)^{2}\left\|x_{n}-x^{*}\right\|^{2}+2 \alpha_{n}\left\langle f\left(x_{n}\right)-f\left(x^{*}\right)+f\left(x^{*}\right)-x^{*}, j\left(x_{n+1}-x^{*}\right)\right\rangle \\
& \leq\left(1-\alpha_{n}\right)^{2}\left\|x_{n}-x^{*}\right\|^{2}+2 \alpha_{n} \alpha\left\|x_{n}-x^{*}\right\|\left\|x_{n+1}-x^{*}\right\|+2 \alpha_{n}\left\langle f\left(x^{*}\right)-x^{*}, j\left(x_{n+1}-x^{*}\right)\right\rangle \\
& \leq\left(1-\alpha_{n}\right)^{2}\left\|x_{n}-x^{*}\right\|^{2}+\alpha_{n} \alpha\left(\left\|x_{n}-x^{*}\right\|^{2}+\left\|x_{n+1}-x^{*}\right\|^{2}\right) \\
& \quad+2 \alpha_{n}\left\langle f\left(x^{*}\right)-x^{*}, j\left(x_{n+1}-x^{*}\right)\right\rangle,
\end{aligned}
$$

which yields that

$$
\begin{aligned}
\left\|x_{n+1}-x^{*}\right\|^{2} \leq & \frac{1-(2-\alpha) \alpha_{n}}{1-\alpha \alpha_{n}}\left\|x_{n}-x^{*}\right\|^{2}+\frac{\alpha_{n}^{2}}{1-\alpha \alpha_{n}}\left\|x_{n}-x^{*}\right\|^{2} \\
& +\frac{2 \alpha_{n}}{1-\alpha \alpha_{n}}\left\langle f\left(x^{*}\right)-x^{*}, j\left(x_{n+1}-x^{*}\right)\right\rangle \\
= & \left(1-\bar{\alpha}_{n}\right)\left\|x_{n}-x^{*}\right\|^{2}+\frac{\alpha_{n}^{2}}{1-\alpha \alpha_{n}}\left\|x_{n}-x^{*}\right\|^{2} \\
& +\frac{2 \alpha_{n}}{1-\alpha \alpha_{n}}\left\langle f\left(x^{*}\right)-x^{*}, j\left(x_{n+1}-x^{*}\right)\right\rangle,
\end{aligned}
$$

where $\bar{\alpha}_{n}=\frac{2(1-\alpha) \alpha_{n}}{1-\alpha \alpha_{n}}$. By boundness of $\left\{x_{n}\right\}$ and condition (i) and Lemma 1.6, $\left\{x_{n}\right\}$ converges strongly to $x^{*}$. This completes the proof of Theorem 2.2.

Remark 2.3. Theorem 2.2 is obtained under the coefficient $\alpha_{n}$ satisfying $\lim \alpha_{n}=$ 0 and $\sum_{n=0}^{\infty} \alpha_{n}=\infty$. In addition, this paper omits the request that space $E$ admits a weakly sequentially continuous duality mapping from $E$ into $E^{*}$. Hence it is an improvement of Theorem 2.4 of [2].

Remark 2.4. If $E$ is uniformly smooth then $E$ is reflexive and has a uniformly Gâteaux differentiable norm with the property that every nonempty closed and bounded subset of $E$ has the fixed point property for nonexpansive mappings(see, remark 3.5 of [9]). Thus, if $E$ is a real uniformly smooth Banach space, then the results in this paper are true, too. 


\section{REFERENCES}

1. S.S.Chang, Some problems and results in the study of nonlinear analysis, Nonlinear Anal., 30(1997) 4197-4208.

2. Y. Song, R.Chen, Viscosity approximation methods for nonexpansive nonself-mappings, J. Math. Anal. Apple., 321(2006)316-326.

3. Tomonari Suzuki, Strong convergence theorems for infinite families of nonexpansive mappings in general Banach spaces, Fixed Point Theory and Applications, 2005:1(2005)103-123.

4. W.Takahashi, Nonlinear Functional Analysis, Yokohama Publishers, Yokohama, 2000.

5. W. Takahashi, Y. Ueda, On Reich's strong convergence for resolvents of accretive operators, J. Math. Anal. Appl. 104(1984)546-553.

6. H.K. Xu, Viscosity approximation methods for nonexpansive mappings, J. Math. Anal. Apple., 298(2004)279-291.

7. H.-K. Xu, Approximating curves of nonexpansive nonself-mappings in Banach spaces, in: Mathematical Analysis, C.R.Acad. Sci. Paris, 325(1997)151-156.

8. Hong-Kun Xu , Iterative algorithms for nonlinear operators, J. London. Math. Soc., 2(2002): $240-256$.

9. Habtu Zegeye, Naseer Shahzad, Strong convergence theorems for a common zero of a finite family of m-accretive mappings, Nonlinear Anal., 66(2007)1161-1169.

1 Department of Mathematics, Honghe university, Mengzi, Yunnan, 661100, China.

E-mail address: zhenhuahe@126.com

2 Department of Mathematics, Hangzhou normal university, Zhejiang, 310036 , CHINA.

E-mail address: gufeng99@sohu.com 